\title{
À l'écoute du métier de communicant public
}

Dominique Mégard et Didier Rigaud

\section{OpenEdition}

Journals

Édition électronique

URL : http://journals.openedition.org/communicationorganisation/3795

DOI : 10.4000/communicationorganisation.3795

ISSN : 1775-3546

Éditeur

Presses universitaires de Bordeaux

\section{Édition imprimée}

Date de publication : 1 juin 2012

Pagination : 171-179

ISBN : 978-2-86781-821-9

ISSN : 1168-5549

\section{Référence électronique}

Dominique Mégard et Didier Rigaud, «À l'écoute du métier de communicant public », Communication et organisation [En ligne], 41 | 2012, mis en ligne le 01 juin 2015, consulté le 30 avril 2019. URL : http:// journals.openedition.org/communicationorganisation/3795; DOI : 10.4000/ communicationorganisation.3795 


\title{
À l'écoute du métier de communicant public
}

\author{
Dominique Mégard', Didier Rigaud²
}

Dans un contexte à forte mutation et à évolution technologique rapide, évaluer l'existant, dresser un état des lieux du métier de communicant public s'imposait comme une nécessité. En décembre 2011, Cap'Com - le réseau national de la communication publique et territoriale - avec la société Occurrence - spécialiste en études et évaluation de la communication - et en partenariat avec le CNFPT - Centre National de la Fonction Publique Territoriale - présente une nouvelle enquête métier. Cette enquête s'inscrit dans la continuité des précédentes menées par Cap'Com depuis le début des années 1990 sur la fonction communication dans les collectivités, soit directement, soit en partenariat avec divers interlocuteurs (dont la revue Public(s) entre 2000 et 2005). Elle permet comme les précédentes, et en comparaison avec leurs résultats, de mieux cerner le profil des communicants territoriaux.

Nous présenterons donc ici les principales conclusions de cette étude dans une perspective évolutive en comparant ses résultats avec des enquêtes similaires antérieures.

\section{Une professionnalisation en mouvement}

La professionnalisation et la reconnaissance des communicants territoriaux sont un phénomène récent apparu durant le milieu des années 1980.

\footnotetext{
1. Dominique Mégard est présidente de Cap'Com, le réseau de la communication publique et territoriale, qui vit et agit sur le terrain de la communication publique depuis 1988. Son dernier ouvrage sur La communication publique et territoriale (collection Topos, éditions Dunod) fait suite à celui écrit avec Bernard Deljarrie sur La communication des collectivités locales (LGDJ - $2^{\mathrm{eme}}$ édition 2009). Chargée de cours en communication publique aux Universités de Paris I Sorbonne, Lille II et l'UCO d'Angers, elle intervient à l'Université Mantouri de Constantine ainsi que dans de nombreux débats ou colloques.

2. Didier Rigaud est spécialiste de la communication publique territoriale. Maître de conférences associé à l'ISIC (Institut des Sciences de l'Information et de la Communication de l'Université de Bordeaux 3), il est consultant associé à l'agence Epiceum (spécialisée en communication publique). Il collabore régulièrement avec différents organismes privés et publics, (CNFPT, Cap'Com, Territorial...) mais aussi depuis plus de 20 ans avec l'ACMQ, l'Association des Communicateurs Municipaux du Québec, province canadienne où il a débuté sa carrière professionnelle.
} 
Auparavant la communication des collectivités résultait, en grande majorité, du travail des élus.

L'exposé du cas de Rennes où le bulletin municipal est fort bien fait mais coûte à la ville $500000 \mathrm{~F}$ par an pour 8 numéros et consomme la plupart des week-ends de M. Janton, l'adjoint au maire chargé de l'information, a permis de poser les questions essentielles, sinon de leur répondre: peut-on réussir un bulletin municipal sans moyens financiers et sans techniciens??

La conjonction de plusieurs facteurs interdépendants va favoriser, au début des années 1980, l'avènement de la professionnalisation de la communication territoriale. Les lois de décentralisation ont pour conséquence de renforcer les collectivités, de faire émerger de nouveaux territoires avec, à leur tête, des élus disposant d'un pouvoir exécutif renforcé. Le contexte de crise économique implique, pour les élus, la nécessité de "rendre compte » de l'utilisation de l'argent public et de communiquer sur les finances et les impôts locaux, sur leur gestion du territoire ainsi que de faire la promotion des services et équipements publics. L'arrivée de nouveaux outils de communication, issus de la télématique, Minitel et journaux électroniques d'information, l'explosion de la vidéo, de la micro-informatique, des radios libres ont diversifié les formes de communication. Avec le vent de la décentralisation, les collectivités éprouvent le besoin de développer une communication identitaire pour se faire connaitre elles-mêmes mais aussi pour attirer des entreprises ou touristes.

Deuxième chance de l'information municipale : elle s'est considérablement professionnalisée. Nous sommes très loin des années 60. C'était le temps sympathique des amateurs, mais il n'empêche que nous observons actuellement et à chaque scrutin local, un accroissement des moyens de communication et l'arrivée de nouveaux professionnels dans le domaine de la communication municipale... Donc nous sommes passés - ceci est important - de l'époque où le maire confiait linformation à un homme de confiance à l'époque où il la confie à un homme "qui sait faire", ce qui est tout à fait différent".

Depuis, le pouvoir et l'impact des collectivités sur la vie et le développement local ont accru la nécessité de "faire savoir ", "faire connaître " et " faire comprendre ». La complexification de la gestion publique locale a transformé la communication en un service dû aux citoyens et en un service public, levier indispensable de l'action publique comme de la participation citoyenne. La communication en s'imposant, s'est professionnalisée davantage, générant des formations initiales et continues de haut niveau tant en universités qu'en écoles spécialisées. Une spécialisation professionnelle s'est créée, imposée,

3. MOSSOLIN C., « La ville de Poitiers organisait les 4 et 5 décembre 1975 un colloque sur le thème de l'information municipale ", Vie Publique, février 1976, p. 11.

4. LANGENIEUX-VILLARD P., " les techniques, les coûts et l'impact de l'information municipale en France », compte rendu des rencontres internationales d'Angoulême, communication locale : nouveaux modes, nouvelles pratiques, organisées par la FMVJ, Fédération Mondiale des Villes Jumelées en mars 1987. 
plus ou moins organisée. Les évolutions du métier de communicant public ont fait l'objet d'observations et d'analyses de tendances dont la dernière est l'enquête métiers présentée ici et qui fait suite à une dizaine d'autres menées ${ }^{5}$ depuis la fin des années 1980 auprès des responsables de communication dans les collectivités.

La meilleure traduction de cette professionnalisation est celle de linscription dans les répertoires des métiers des 3 fonctions publiques, de métiers clairement recensés correspondant à des savoir-faire et des connaissances bien identifiés (...) Dans le cadre du répertoire des 231 métiers territoriaux (établi par le CNFPT, centre national de la fonction publique territoriale) 6 métiers de la communication sont recensés dans le cadre de la filière administrative, l'une des huit filières reconnues ${ }^{6}:$ directeur de la communication; chargé de communication (en tous genres y compris chargé de relations presse ou chargé de l'événementiel); chargé de publication (appellation qui remplace celle de journaliste, les critères de définition de cette dernière profession n'étant pas remplis dès lors que l'on travaille dans une collectivité); créateur de support graphique et audiovisuel; chef de projet multimédia et photographe-vidéaste. Ces métiers, ainsi que les savoirs et les compétences associés, ont été définis par une commission au début des années 2000 et ont donné lieu à des discussions acharnées tant se levaient de difficultés à conjuguer raisonnements administratifs et réalité communicationnelle. Cette recension dans les répertoires n'est pas exhaustive des fonctions existant au sein des services communication dans les collectivités ou autres organismes publics. Elle n'a, en effet, aucun caractère obligatoire et reste un outil d'accompagnement et d'aide à la décision en ressources humaines. Mais elle est significative et intéressante en terme de reconnaissance d'une fonction communication qui questionne et se questionne sur sa place au sein des organisations. Les questions et remises en question demeurent en effet nombreuses. La communication (et donc les compétences qui y sont associées) n'est par exemple, toujours pas reconnue comme option possible des concours administratifs?

\section{Cartographie d'une profession : enquête métier 2011}

L'enquête 2011 a été auto-administrée par Internet et lancée auprès de 5000 communicants de collectivités locales. Le questionnaire a été mis en ligne du 10 octobre au 14 novembre 2011. Au total, 588 communicants ont répondu.

\section{Des professionnels chevronnés}

Les communicants ont un haut niveau de formation dans l'ensemble. En 2011, près des deux tiers des communicants publics ont un niveau d'études supérieur à bac +4 dont $39 \% \mathrm{bac}+5$ et plus. Cette tendance va sans doute se renforcer dans les années à venir, dans la mesure où les nouveaux arrivants

5. Par Public(s) et le réseau Cap'Com ou en association avec lui.

6. Les autres filières : technique; culturelle; sportive ; sanitaire et sociale; sécurité ; animation et sapeurs-pompiers. Au sein de ces filières sont recensés 55 cadres d'emplois. Chacun regroupe des agents dont les emplois et les métiers ont des caractéristiques professionnelles proches.

7. In MÉGARD D., La communication publique et territoriale, Dunod Topos, 2012. 
dans la profession sont plus diplômés que leurs aînés (74 \% des moins de 35 ans ont un niveau bac+4 et plus contre $55 \%$ des plus de 45 ans).

En 1995, une enquête réalisée auprès de 154 responsables de communication des villes de plus de 10000 habitants, départements et régions par l'association Melting Com et La Gazette des communes donnait les résultats suivants ${ }^{8}$ : $49,3 \%$ disposaient d'un diplôme à bac+4 et plus dont $22,7 \%$ à bac +5 et plus.

En 2000, une nouvelle étude Public(s), Cap'Com et Progress ${ }^{9}$ (échantillon de 166 responsables de communication de collectivités de plus de 20000 habitants, enquête par questionnaire réalisée du 28 juin au 16 août 2000) révélait une évolution sensible par rapport à 1995 puisque $67 \%$ des communicants publics étaient titulaires d'un diplôme à bac +4 ou plus.

En terme de branche, les diplômes en communication montent en puissance au détriment des sciences humaines et du droit.

En 2011, 44 \% des communicants territoriaux possèdent un diplôme en communication (contre $37,7 \%$ en $1995^{10}$ ), $28 \%$ en sciences humaines et politiques, $10 \%$ en commerce, gestion, économie ou marketing et $7 \%$ en ressources humaines, management ou droit.

Graphique : source Occurrence

Dans quelle discipline avez-vous obtenu votre diplôme le plus élevé ?

Communication
Sciences Humaines

\section{Le profil type du communicant public}

La communication est un secteur réputé comme étant plus féminisé que d'autres, notamment dans la communication des collectivités. Les femmes

8. BINOT J.-M., « Enquête Melting Com-La Gazette. Directeur de la communication : portrait d'une profession », La Gazette, n' 1294, 23 janvier 1995, p. 13.

9. « La communication locale, un vrai métier », Publics, n¹1, octobre 2000, p. 3.

10. Op. cit., p. 2. 
Sexe :

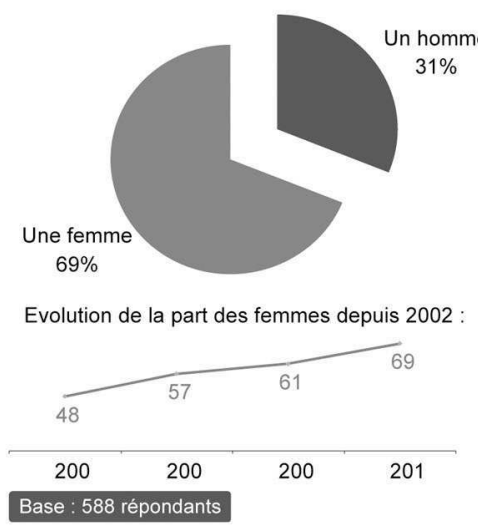

Age :

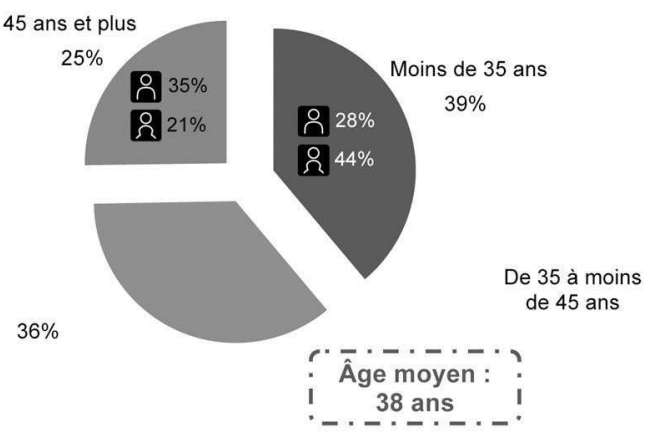

représentent aujourd'hui plus des deux tiers des effectifs, soit $69 \%$, un chiffre qui a dépassé le taux de féminisation de l'ensemble de la fonction publique territoriale $(61 \%)$. Toutefois les femmes n'occupent que $59 \%$ des postes de directeurs de la communication.

Cette augmentation de la féminisation est constante durant ces 10 dernières années, $48 \%$ en 2002, $57 \%$ en 2005 et $61 \%$ en $2008^{11}$.

Lâge moyen du communicant territorial est de 38 ans, $39 \%$ ont moins de 35 ans et $26 \%$ plus de 45 ans. En 1995, 36,7\% avaient moins de 35 ans, $37 \%$ en 2000 et 46,3\% en 2005. La comparaison de ces chiffres avec la pyramide des âges de la fonction publique territoriale (FPT), nous confirme que la communication territoriale est une profession jeune. Un état qui contraste fortement avec certaines filières professionnelles, l'âge moyen en FPT, tous métiers confondus, étant 42 ans.

Enfin, notons que parmi les moins de 35 ans, environ les trois quarts sont des femmes, le processus de féminisation n'étant certainement pas terminé.

\section{Disparité et stagnation des rémunérations}

La moitié des communicants publics $(51 \%)$ ont une rémunération annuelle brute (primes comprises) inférieure à 30000 et $20 \%$ seulement une rémunération supérieure à 40000 . Le salaire médian d'un directeur de communication au sein d'une collectivité territoriale se situe entre 35000 et 40000 , celui d'un responsable de communication autour de 30000 et celui d'un chargé de communication entre 20000 et 25000 .

En 2005 « les panélistes sont 60,6\% à percevoir un traitement annuel brut inférieur à 29000 . Classiquement, les femmes, a fortiori si elles sont jeunes, apparaissent défavorisées. $26,7 \%$ des interrogées gagnent moins de 20000 par an contre $13,4 \%$ des hommes $» .{ }^{12}$ Cette disparité de rémunération

11. Source Cap'Com, « enquête métier » 2011.

12. Op. cit., p. 2. 
est certainement l'explication à cette relative stagnation du niveau des rémunérations outre le contexte économique global.

À noter : la rémunération arrive en dernière position (2\%) des raisons qui ont attiré vers la communication territoriale loin derrière le goût pour la vie locale (47 \%) ou l'attachement au service public (37\%).

\section{Un métier en constante évolution}

La communication territoriale est " poreuse » et de nombreuses passerelles existent entre public et privé voire entre les différentes fonctions ou organismes publics, hors collectivités... Près de la moitié des communicants territoriaux ont exercé leur métier ailleurs qu'en collectivité, $41 \%$ dans le privé et $11 \%$ dans une autre fonction publique. $49 \%$ ont exercé un autre métier, comme par exemple, journaliste, attaché parlementaire, directeur de cabinet, enseignant, fonction marketing ou ressources humaines, etc.

Les études précédentes révélaient également cette diversité d'activités antérieures à celle de communicant. "Les qualités jugées nécessaires au bon exercice de leur fonction par les directeurs de la communication ne sont pas spécifiques à la communication : ainsi sont placées en têtes les qualités relationnelles, la polyvalence et la capacité d'organisation tandis que la culture politique, les qualités rédactionnelles et la créativité sont citées en dernier $»{ }^{13}$

La moitié des communicants occupent leur poste actuel depuis moins de 5 ans et seulement $10 \%$ depuis 10 ans ou plus. La fonction communication au sein des collectivités a toujours été caractérisée par un « turn-over » important lié à sa dimension stratégique et politique.

Les métiers de la communication conservent des perspectives de carrières intéressantes pour $80 \%$ des communicants territoriaux. Un résultat en hausse par rapport à 2008 (75\%). La perspective de pouvoir continuer d'exercer ce métier au sein du secteur public reste aussi forte en 2011 qu'en 2008, 75 \%, mais plus importante qu'en 1995, $63 \%{ }^{14}$

Les communicants territoriaux restent séduits par le secteur privé où les rémunérations sont plus attractives. Ils s'orientent facilement vers des agences ou les métiers du consulting.

Plus des trois quarts des communicants territoriaux (79 \%) ont suivi une formation au cours des trois dernières années pour suivre les évolutions des outils et technologies mais aussi pour accompagner un changement ou une évolution de poste, les besoins se situant alors plus dans le domaine du management et de la stratégie.

13. «La communication locale, un vrai métier ", Publics, n¹1, octobre 2000, p. 3.

14.Binot J.-M., «Enquête Melting Com-La Gazette. Directeur de la communication : portrait d'une profession », La Gazette, n¹294, 23 janvier 1995, p. 14. 


\section{Qualités requises et motivations}

Les communicants du panel affirment à $47 \%$ que le goût pour la vie locale a motivé leur choix professionnel de travailler au sein d'une collectivité. Viennent ensuite, l'intérêt pour les enjeux politiques, $39 \%$, et leur attachement au service public, $37 \%$. Seuls $10 \%$ citent la sécurité de l'emploi.

Enfin, les qualités que les communicants territoriaux jugent indispensables de posséder sont : " disposer d'une vision stratégique " $(73 \%)$, " maitriser les techniques de communication » (59\%), « avoir le sens politique » $(41 \%)$, « avoir une bonne connaissance du tissu local » $(41 \%)$, « savoir manager » (37\%) et « être capable d'anticiper » $(33 \%)$.

\section{Une multiplicité de missions face à des moyens limités}

On ne constate pas d'augmentation des attributions des services communications, les principales sont : l'information des publics (magazines, journaux, lettres d'informations, $97 \%$ ), les relations de presse (89\%), l'événementiel (85\%), les technologies del'information et de la communication $(68 \%)$, la communication interne $(65 \%)$, les relations publiques et le protocole (58\%), la communication des élus (rédaction des discours et éditoriaux) (50\%), le marketing territorial et la promotion du territoire ( $47 \%)$, la documentation et la veille médiatique (38\%), la démocratie participative $(22 \%)$ et enfin, l'accueil des publics ( $20 \%$ ).

Les communicants publics ont le sentiment pour la grande majorité d'entre eux (83 \%) que leurs missions ne cessent d'augmenter, les nouveaux outils, supports et actions s'ajoutant à ceux existants alors que les ressources humaines et budgets restent globalement stables. Les services communication des collectivités locales sont constitués pour $40 \%$ d'entre eux de moins de 3 personnes, l'effectif moyen est de 8 personnes dans les communes de plus de 5000 habitants, 7 pour les intercommunalités et 17 pour les conseils généraux et régionaux.

$76 \%$ des communes ont réduit leur budget consacré à la communication ainsi que $91 \%$ des conseils généraux et régionaux ; les intercommunalités ont bénéficié pour $39 \%$ d'entre elles d'une hausse de leur budget communication.

Au niveau des effectifs de ces services, le constat est le même. Ils sont en baisse pour $77 \%$ des communes, $81 \%$ des conseils généraux et régionaux alors que les structures intercommunales ont vu les effectifs de leurs services communication croître pour $37 \%$ d'entre elles.

On pourrait en déduire qu'un transfert de la gestion de la communication s'opère entre les communes et le niveau supra-communal. Même si on peut constater des fusions de services dans certaines agglomérations, force est de constater que la mise en place d'une communication « concertée » sur ces « nouveaux territoires » et loin d'être généralisée. "Dans seulement $13 \%$ des communes, la concertation avec la communauté sur les questions de communication est permanente. Pour $25 \%$ elle ne se fait que sur les projets communs. Pour 
$63 \%$ elle est soit occasionnelle, soit inexistante. Les communautés reconnaissent aussi cette faible concertation. $41 \%$ des communautés avouent quien matière de communication la concertation n'est qu'occasionnelle ou nulle avec les communes de leur propre territoire ${ }^{15}$ ».

\section{L'adaptation à la digitalisation}

Le baromètre 2011 «Les Français et la communication locale » a confirmé la tendance des résultats de l'enquête précédente de 2009, à savoir la forte audience par la population des canaux d'information locale avec un glissement des outils traditionnels vers les supports digitaux : $95 \%$ des Français utilisaient au moins un support papier pour s'informer sur la vie locale et $66 \%$ au moins un support digital.

Cette tendance est perçue et anticipée par les communicants publics. D'après eux, le management des réseaux sociaux et le marketing territorial sont deux compétences qui se développeront dans les années à venir.

\section{Conclusion}

Si cette étude quantitative de la profession ne répond en rien à toutes les problématiques liées au métier de communicant public, par ailleurs largement développées dans ce numéro de la revue, elle permet, toutefois, d'appréhender le positionnement de la communication dans l'organisation territoriale. Questionnement récurrent pour les communicants territoriaux : quel positionnement avoir par rapport aux élus, à l'administration ? Quel statut? Que faire en situation de changement politique? La question du rattachement du service par exemple caractérise et symbolise tout à la fois la réalité et la complexité de la communication territoriale. Rattachée au Directeur Général des Services (DGS), la direction de la communication sera "banalisée ", considérée comme une direction comme les autres. Rattachée directement à l'exécutif ou au cabinet, elle sera considérée comme une fonction plus politique. Sur cette question, les rattachements directs au politique (maireprésident ou cabinet) sont majoritaires (de 47,5\% rattachement au maire et $21 \%$ au cabinet en 2000 à $36 \%$ au maire et $29 \%$ au cabinet en 2011). Mais avec dans le même temps, une explosion des rattachements (souvent par le jeu du double rattachement) au DGS (23,5\% en 2000 à $51 \%$ en 2011).

\section{BIBLIOGRAPHIE}

DELJARRIE B., MÉGARD D., avec la participation de RIGAUD D., La communication des collectivités locales, Paris, LGDJ- Lextenso, $2^{\text {ème }}$ édition, 2009.

15. Cap'Com, « enquête métier » 2011. 
HARTEREAU A., La communication publique territoriale, Paris, éditions du CNFPT, 1996.

LE NET M., La communication publique : pratique des campagnes d'information, Paris, La documentation française, 1993.

LORANT G., Les collectivités locales face aux défis de la communication, Paris, L'Harmattan, 2005.

MÉGARD D., La communication publique et territoriale, Dunod, Paris, 2012.

MESSAGER M. (pseudonyme pour ouvrage collectif), La communication publique en pratique, Paris, 1995.

SOUCHARD M., WAHNICH S., La communication politique locale, Que sais-je ?, Paris, 1995.

ZÉMOR P., La communication publique, Paris, PUF, 1995.

Résumé : Aujourd'hui, environ 15000 personnes exercent une véritable fonction de communication, interne ou externe, au sein des collectivités locales. Leurs missions sont de plus en plus importantes. Cet article dresse, à partir de l'enquête métier Cap'Com de 2011, le portrait de ces communicants.

Mots-clés : communication publique territoriale, profil communicant.

Abstract : Today, approximately 15000 persons exercise a real office of communication, internal or external, within local authorities. Their missions are more and more important. This article draws up, from the investigation job of Cap'Com 2011, the portrait of these communicators.

Keywords :Territorial public Communication, Communicating profile 
